\title{
Fertility preservation options for children and adolescents with cancer
}

\author{
Rodrigo L.P. Romao, MD; ${ }^{\prime}$ Armando J. Lorenzo, MD, $M S c^{2}$
}

'Division of Pediatric Urology and Pediatric General Surgery, IWK Health Centre, Dalhousie University, Halifax, NS, Canada; '2Division of Pediatric Urology, The Hospital for Sick Children and Department of Surgery, University of Toronto, Toronto, ON, Canada

Cite as: Can Urol Assoc J 2017;1 1 (1-2Suppl1):S97-102. http://dx.doi.org/10.5489/cuaj.4410

\section{Abstract}

Introduction: Cancer in children and adolescents has seen a stark rise in survival rates in the last decades; overall survival in excess of $80 \%$ can be reasonably expected for many newly diagnosed patients with malignancies in this age group. Survivorship has unfolded several specific issues faced by these patients, including fertility concerns. Hence, fertility preservation efforts have been discussed and undertaken with increased frequency.

Methods: In this article, the authors provide a broad overview of the current recommendations surrounding fertility preservation in this patient population. Reasons to offer fertility preservation, target groups for interventions, and methods available based on age group and gender are discussed in detail.

Results: The medical literature and patient advocates strongly support a discussion about fertility preservation at the time of diagnosis; the risk of infertility is real and parents and families wish to be informed about it. In postpubertal males, sperm-banking is relatively straightforward and should be attempted by most newly diagnosed patients, ideally before commencement of treatment. Cryopreservation of testicular tissue in higher-risk prepubertal males is feasible, but still experimental. Female fertility preservation is more complex, requires more invasive procedures, and can delay initiation of treatment due to the requirement for hormone stimulation of follicles prior to harvesting.

Conclusions: Oncofertility initiatives in children and youth are still in their early days and will continue to expand; urologists should be prepared to offer counselling and interventions when appropriate to this growing vulnerable population.

\section{Introduction}

Many pediatric cancers are associated with a favourable prognosis compared to their adult counterparts; cure rates around $80 \%$ can be reasonably expected in the developed world for the majority of cases. ${ }^{1}$ Success in therapy has translated into patients growing up and dealing with the aftermath of treatment, including sequelae from surgery, radiation, and chemotherapy. Indeed, the wide array of medical and social issues affecting adolescent and adult survivors of pediatric cancer have turned this aspect of care into a subspecialty in and of itself. Survivorship primarily tackles issues such as long-term toxicity from chemotherapy and radiation, consequences of extirpative surgery, and monitoring and treatment of secondary malignancies, to name a few. Nonetheless, mental health and social aspects - such as the desire of survivors to exert a meaningful societal role and concerns regarding their ability to start a family — are also at the core of this multifaceted discipline. ${ }^{2}$ The potential negative gonadotoxic impact of treatments offered in childhood and adolescence have placed fertility preservation (FP) for children, adolescents, and young adults with cancer at the forefront of survivorship efforts.

Table 1 summarizes the aspects discussed below, i.e., reasoning behind $\mathrm{FP}$ for cancer patients, groups to be targeted, and the timing of interventions.

\section{Why discuss fertility preservation for children and adolescents with cancer?}

There are multiple ways fertility may be affected in cancer patients. This includes adverse impact from the tumour itself (as seen in some cases of testicular neoplasms), gonadotoxic effect of medications or radiation, impact on the hypothalamic-pituitary-gonad axis (related to hormone production), and disruption of normal anatomical pathways to ensure appropriate gamete delivery, fertilization, and implantation. For example, many alkylating agents, such as cyclophosphamide and ifosfamide, are notorious for causing transient or permanent azoospermia. ${ }^{3}$ Although toxicity follows a dose-dependent pattern, no safe cutoff below which recovery is guaranteed has been identified. ${ }^{4}$ These agents are regularly used in the treatment of brain tumours, leukemia, neuroblastoma, sarcomas, and others. 


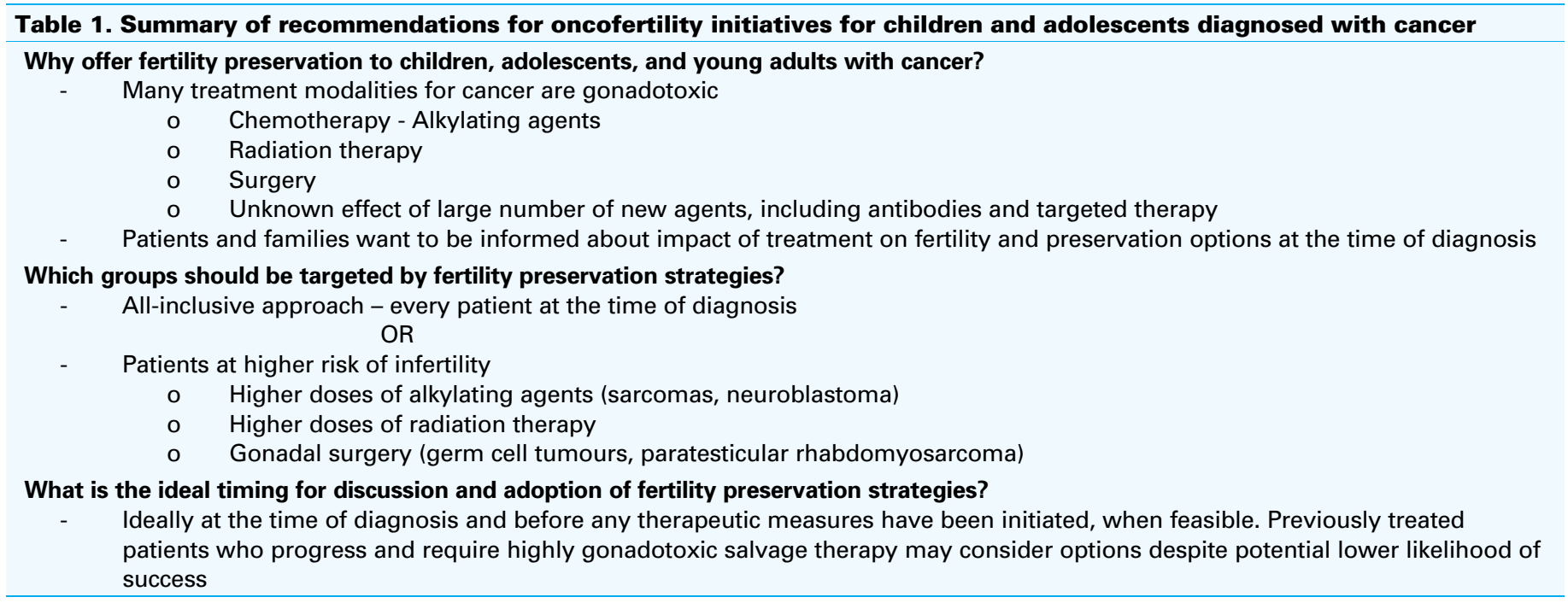

Radiation therapy is another example of a gonadotoxic treatment frequently employed in children and adolescents, with direct or indirect doses leading to temporary or permanent azoospermia. ${ }^{5}$ Moreover, radiation can affect reproductive organs, such as the uterus, with subsequent issues related to implantation and carrying a pregnancy. In addition to damage leading to decreased or absent sperm counts, effects can have a long-lasting effect on DNA integrity, thus reducing the quality and quantity of gametes. Moreover, the impact of gonadotoxic interventions may be different in children due to differences in developmental stage (i.e., prepubertal state), as well as potential worse impact on DNA by affecting stem and precursor cells. Unfortunately, when it comes to the long-term impact of current interventions, it is often an unknown estimate due to lack of reliable long-term data with modern multimodal therapy.

In addition to the aforementioned biological plausibility, there is evidence that cancer treatment in childhood and adolescence leads to infertility. In a report from the Childhood Cancer Survivor Study, the risk of infertility was 2.5 times higher in male cancer survivors compared to healthy siblings ( $46 \%$ vs. $17.5 \%$ ); on multivariate analysis, higher alkylating cumulative dose, surgical excision of an organ of the genital tract, testicular radiation $\geq 4 \mathrm{~Gy}$, and exposure to bleomycin were identified as independent risk factors for infertility. ${ }^{6}$

Despite the paucity of information and reliance on extrapolations or theoretical concerns, cancer survivors and their families value a frank discussion about fertility at the time of diagnosis. At first glance, one might wonder why even bother bringing up the issue in the dramatic setting of disclosing a cancer diagnosis and the pressing need to start treatment for a patient that is far from planning to have a family of his or her own. In a multicentre Canadian study looking at the perspectives of survivors, parents, and provid- ers, Gupta et al found that parents and survivors would like to be informed at the time of diagnosis, regardless of the actual risk of fertility impairment, and despite other factors, such as cost, experimental nature of interventions, and likelihood of surviving. Furthermore, non-disclosure was noted to be associated with future negative feelings, such as resentment and anger.'

Concerns regarding parenthood can have important implications to survivors of childhood cancer, some that go beyond the biological ability to have a child. Infertility and subfertility can lead to negative psychological outcomes not only due to its significance as a standalone issue, but also secondary to its perceived negative impact on romantic relationships. ${ }^{8}$ Therefore, concerns, anxiety, and embarrassment due to the impact of therapy on sexual and reproductive function have the potential to adversely impact quality of life. Fertility preservation discussion may help alleviate some of these adverse psychological outcomes. Moreover, long-term assessment becomes more comprehensive, considering underappreciated aspects, such as body image and difficulties with intimacy.

These views are in line with modern statements from major organizations dealing with cancer diagnosis, management, and prevention. For example, the American Society of Clinical Oncology recently updated guidelines on FP, acknowledging that adults, adolescents, pediatric patients and their families, and cancer survivors should be informed of FP options. The guidelines clearly state that a frank discussion about infertility risks and options for preservation should occur as early as possible, and, if feasible, before treatment starts. ${ }^{9}$

There are salient differences and discrepancies noted in the literature and clinical care. This is highlighted in a systematic review of existing clinical practice guidelines on this subject. Font-Gonzalez et al identified 25 guidelines, 
of which eight were considered of higher quality based on an instrument for appraisal of clinical guidelines that evaluates six domains. Among the eight higher-quality guidelines, there was high discordance in some of the recommendations for male FP, such as to which patients FP strategies should be offered, by which healthcare professionals, timing, and methods of collection. ${ }^{1}$ As we move forward, it is critical that recommendations become more homogeneous and evidence-based, yet open to the limitations imposed by lack of data and time lag between exposure (treatment) and desire to have a family. Some of these controversies will be addressed in more detail.

\section{Which patients should be offered fertility preservation?}

There is no unified consensus defining which groups of young patients with cancer should be offered FP. Some favour an all-inclusive approach, where FP is offered to every single newly diagnosed postpubertal cancer patient based on the premise that at the time of diagnosis, it is impossible to accurately predict who will go on to achieve cure with minimal toxicity vs. relapse with need for further treatment of incremental gonadotoxic potential. This latter scenario is not uncommon and can generate difficult discussions around FP, as the patient has been exposed to chemotherapy and radiation, yet is bound to be exposed to therapies that dramatically increase the risk of infertility. For example, patients with relapsed leukemia requiring bone marrow transplant with whole-body irradiation are in a more severe gonadotoxic category than a remitting leukemia that progresses to cure with standard therapy. Obviously, the principle in this approach is to attempt preservation in many, knowing that it will potentially be unnecessary for a significant number, with the psychological and financial implications it carries.

Conversely, others endorse a more selective approach, where only high-risk patients are targeted. This would include patients diagnosed with cancers known to require exposure to known gonadotoxic agents (such as high doses of alkylating agents, as seen in cases of rhabdomyosarcoma, Ewing's sarcoma, neuroblastoma, Hodgkin's lymphoma) or radiation to achieve cure (e.g., patients undergoing totalbody irradiation prior to bone marrow transplantation), or else tumours directly affecting the gonads (testicular germ cell tumour or paratesticular rhabdomyosarcoma).

The discussion is undoubtedly complex and fueled by strong views on both sides. The all-inclusive approach is appealing, given the unpredictability of cancer outcomes at the time of diagnosis and the limited tools available to reliably counsel patient and families on the risk of relapse with an unforeseen requirement for further gonadotoxic treatments. On the other hand, FP demands use of limited resources, incurs an important upfront and yearly cost that, in many settings, is covered by families. Hence, it may not be necessarily cost-effective, particularly for families that are financially constrained. The more selective approach surely offers a more cost-effective strategy by focusing on populations at higher risk; nonetheless, it may exclude members of the low-risk patient population that ultimately face a more challenging road towards cure with added long-term side effects (including infertility) due to therapy intensification. Our view is that the best estimate of the risk of infertility should be discussed openly with every single newly diagnosed young patient with cancer and all options for preservation presented, empowering patients and families to have the final say on the matter.

\section{Which member of the healthcare team should be made responsible to discuss fertility preservation with patients and families, and what is the best timing for discussion?}

In a mixed-methods systematic review, Vindrola-Padros et al identified several barriers related to healthcare professionals' ability and comfort with discussing fertility issues with their patients and families. The main areas for improvement identified were knowledge gaps on fertility, lack of educational materials, sense of embarrassment and/or discomfort discussing the topic in view of specific patientrelated factors, such as poor prognosis or adverse financial situation, or personal cultural barriers or beliefs. ${ }^{10}$ While in most centres the responsibility for this discussion falls to the oncology team, it appears reasonable to have a dedicated team with professionals that specifically focus on this aspect of care. Critical to this approach are the inclusion of an advance practice nurse, nurse coordinator or nurse practitioner, and the creation of decision tools and educational materials to facilitate presentation of the topic. The development of a multidisciplinary oncofertility group with participation of nurses, oncologists, urologists, gynecologist, ethicists, administrators, and reproductive specialists is also recommended and can assist with implementation of best practices and counselling, being mindful of cultural issues, financial constraints, and individual preferences and beliefs. ${ }^{11}$ Furthermore, this field offers an opportunity for engagement of pediatric urologists and infertility specialists who share an interest in oncology.

In terms of timing, most authors agree that ideally FP should take place before the initiation of chemotherapy and/ or radiation. This sets a relatively tight timeframe, as following confirmation of the diagnosis and appropriate staging, there is a pressing need to start therapy as soon as possible. There are some cases in which therapy has to be started right away and delaying interventions to explore FP may be ill-advised, as it may compromise survival or add morbidity. 
This includes patients with cord compression and pulmonary embolism, among others. A balance may be difficult to achieve, as deferring discussion and exercising FP options after therapy has begun may adversely impact the quality of the tissue. Many treatments (even some that are not permanently gonadotoxic) can trigger transient changes in in gametocyte DNA, along with changes in number and function. Hence, collection before any exposure to cancer treatments is viewed as optimal. ${ }^{9}$

\section{Methods for male fertility preservation}

In postpubertal males, semen cryopreservation is a safe and reliable method for FP. Although obtaining a sample through masturbation may sound straightforward, many patient-related barriers will preclude a successful collection of a sperm sample. There are important cultural and religious considerations at play. Moreover, anxiety and stress triggered by the demand to produce a sample for banking can make it difficult or impossible for a young man to comply. Conceptually, patients close to pubertal development may experience a greater challenge. The yield of banking via masturbation decreases as the patient is early in the pubertal developmental stage. ${ }^{12}$ Other issues to consider are limitations imposed by the primary disease (such as disruption of normal neurological pathways for ejaculation, as seen in patients with cord compression), and side effects of analgesics (such as narcotics required in cases of difficult pain control). Sperm quantity and quality is also adversely impacted by the primary tumour, most notably in cases of malignant testicular neoplasms.

The overall success rate of sperm-banking in this population is not well-known. In a large, multicentric study from a sperm-banking network in France, over 4300 young cancer patients visited a fertility clinic and $93 \%$ were able to provide a sample; $3616(83 \%)$ provided enough material to allow sperm to be cryopreserved. ${ }^{12}$ A study from the U.K. with smaller numbers (180 patients) yielded less reassuring results, with only $66 \%$ of the patients being able to bank sperm and azoospermia found in $13 \% .{ }^{13}$ Although the French authors demonstrate an encouraging yearly increase in referrals for sperm-banking that approached $10 \%$ per year between 1973 and 2007, ${ }^{12}$ the true denominator is not known; it is likely that the number of patients seeking sperm-banking will continue to increase worldwide, as awareness heightens and the proper counselling is put in place. In other words, the data show that once patients get referred through the appropriate channels, there is a good chance that a viable sample will be generated and preserved.

Reasons for failing to provide a sperm sample are many. ${ }^{8}$ First, the distress caused by a recent cancer diagnosis coupled with the pressure of having to obtain a sample quickly before the start of treatment constitute a heavy burden for young teenagers. Second, some patients can be significantly ill and unwell, therefore, physically unable to provide a sample. Third, some young teenagers might not have enough knowledge about masturbation or cultural values may hinder their ability to masturbate. Fourth, it has been shown that teenagers have a difficult time providing a sample when their parents are in the waiting room; ${ }^{8}$ creating a friendly environment to allow a successful collection is not logistically simple.

When masturbation is not a viable option, other methods are available for obtaining a semen sample. Electroejaculation, a method often employed in adult patients with neuropathic dysfunction, can be offered to peri and postpubertal males (Fig. 1). This, however, would require general or regional anesthesia, and education to accept the need to use a rather large rectal probe to stimulate emission and ejaculation. Given that most teenagers diagnosed with cancer will undergo a general anesthetic shortly after diagnosis for procedures such as placement of a long-term vascular access device, bone marrow biopsies, and lumbar puncture with administration of intrathecal chemotherapy, electroejaculation could easily be done concurrently in patients who fail to provide a sample through masturbation. The published experience with this technique in the adolescent age group is still limited, but success rates close to $50 \%$ have been reported..$^{14}$

If electroejaculation fails to produce a sample with sperm or in patients unwilling or unable to tolerate the procedure, direct recovery from the testicle or epididymis can be performed. In general, younger males undergo direct retrieval from the testicle, via aspiration or, more commonly, biopsy (i.e., testicular sperm extraction [TESE]).This is also a good option for patients who produce a sample with azoospermia or severe oligospermia. It is obviously more invasive and can be associated with lower sperm retrieval rates when compared to quantity and quality of sperm obtained via masturbation. In a comparative study, Berookhim et al reported a $60 \%$ success rate for electroejaculation and a $33 \%$ success rate for TESE when assisting 49 patients with a diagnosis of

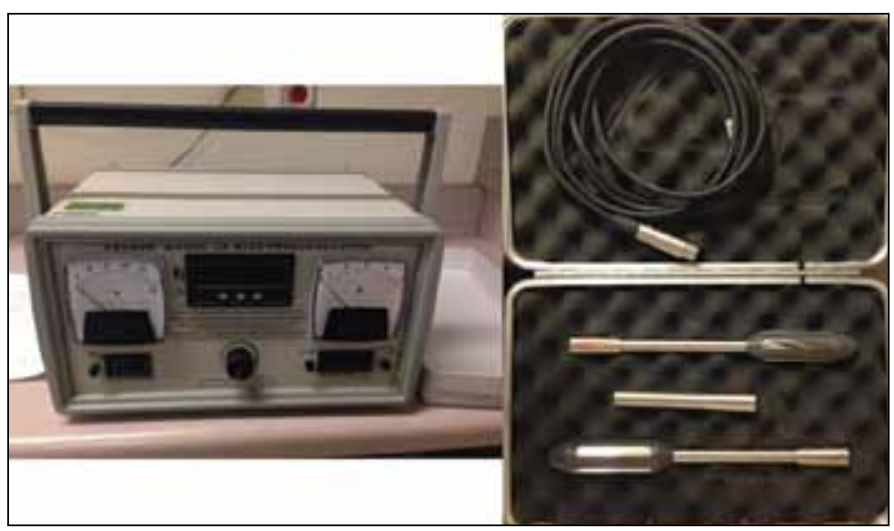

Fig. 1. Electroejaculation machine and probes. 
cancer that were unable to provide a sample for cultural/ religious reasons or who had oligo/azoospermia. ${ }^{15}$

\section{Prepubertal boys}

Puberty represents an important developmental milestone for FP. In males, pubertal changes forecast the maturation of germinal epithelium, with progression towards spermatids and mature sperm. Before puberty, any retrieval option is unlikely to produce cells that can be currently employed for assisted reproductive techniques. Therefore, based on available technology, there is no value is harvesting testicular tissue in prepubertal boys, irrespective of their infertility risk.

This view can be considered as short-sighted. As previously mentioned, the lag time between exposure to cancer treatment and desire to father a child can be in the order of decades. As such, it is likely that technological advances will allow in vivo or in vitro maturation, or novel assisted reproductive techniques generate options currently unavailable. Indeed, Yokonishi et al reported successful pregnancy in mice using neonatal testicular tissue with spermatogenesis induced in vitro; ${ }^{8}$ healthy offspring were produced and were further able to produce progeny of their own. ${ }^{16}$ Thus, many centres around the world consider or offer testicular tissue cryopreservation prior to exposure to chemotherapy. Although conceptually appealing, important ethical, logistical, and financial issues arise, including the need for further research in order to make clinical use of prepubertal tissue a reality. Thus, tissue allocation for research should be considered during the process of harvesting. Moreover, the costs incurred can be significant, representing an option that is sometimes hard to support with the lack of clinically feasible options for tissue maturation in the near future. Nevertheless, research shows that parents and survivors are interested in experimental options, demand to be informed, and involved in the decision-making process. ${ }^{17}$

The procedure itself is straightforward, and not particularly different from similar interventions done in postpubertal males. It can be coordinated with other interventions that require a general anesthetic, adding on average less than 15 minutes to the total operating room time. It is difficult to estimate the amount of tissue to sample, yet it is critical to consider that total testicular volume in the prepubertal age group is rather limited. For most patients, a $3-5 \mathrm{~mm}$ incision of the tunica albuginea allows for removal of 3-4 small (1-2 $\mathrm{mm}^{3}$ ) specimens, which are placed on media and immediately transferred to the bank for processing and storage.

Methods for male FP are summarized in Table 2.

\section{Female patients}

The field of FP has advanced dramatically in terms of options for female patients, and options are better offered in consultation with the team's gynecologists. Harvesting oocytes in postpubertal females can be challenging and has to be timed appropriately with any interventions aimed at hormonal stimulation; the latter necessarily delays the start of treatment by at least two weeks, which can be prohibitive with some pediatric malignancies. A growing body of evidence supports strategies that have been implemented in young adult women with malignancies such as breast cancer. Younger patients are unlikely to tolerate many of the required investigations and interventions, which are customarily done transvaginally. In these, as well as in prepubertal females, oophorectomy or resection of ovary strips can be considered based on parental/patient desire and infertility risk. In girls, urological support in the operating room may be of value, considering that pediatric urologist do many procedures that involve laparoscopic evaluation and manipulation of gonads (i.e., diagnostic laparoscopy and laparoscopic orchidopexy). Close collaboration with pediatric surgeons and gynecologists is critical to expand these options to this patient population.

\begin{tabular}{|c|c|c|c|c|}
\hline \multirow{4}{*}{$\begin{array}{l}\text { Age } \\
\text { Postpubertal }\end{array}$} & \multicolumn{2}{|c|}{ Modality } & \multirow{2}{*}{$\begin{array}{c}\text { Indications } \\
\text { All newly diagnosed patients } \\
\text { vs. higher-risk group }\end{array}$} & \multirow{2}{*}{$\begin{array}{l}\text { Barriers } \\
\text { Inability to produce a sperm sample sue to } 1 \text { ) cultural/ } \\
\text { personal issues (anxiety); } 2 \text { ) patient clinically unwell }\end{array}$} \\
\hline & \multirow[t]{2}{*}{$\begin{array}{c}\text { Sperm } \\
\text { cryopreservation }\end{array}$} & Masturbation & & \\
\hline & & Electroejaculation & $\begin{array}{l}\text { Patient is unable to produce a } \\
\text { sample through masturbation }\end{array}$ & $\begin{array}{l}\text { Requires specialized equipment and general } \\
\text { anesthesia }\end{array}$ \\
\hline & $\begin{array}{l}\text { Direct sperm } \\
\text { extraction } \\
\text { (TESE) or tissue } \\
\text { cryopreservation }\end{array}$ & Testicular biopsy & $\begin{array}{l}\text { Aforementioned methods } \\
\text { have failed }\end{array}$ & $\begin{array}{c}\text { Requires general anesthesia and minor surgical } \\
\text { procedure } \\
\text { Lower success rates }\end{array}$ \\
\hline Prepubertal & \multicolumn{2}{|c|}{$\begin{array}{l}\text { Testicular biopsy with tissue } \\
\text { cryopreservation }\end{array}$} & Experimental & $\begin{array}{l}\text { Requires general anesthesia and minor surgical } \\
\text { procedure } \\
\text { Ethical issues around consent, need for research, } \\
\text { experimental nature, and uncertainty of long-term } \\
\text { feasibility }\end{array}$ \\
\hline
\end{tabular}


Romao et al.

\section{Ethical issues}

There are important ethical connotations to FP. Procurement of cells or tissue for reproductive purposes happens at a difficult time and decisions are accelerated by pressures imposed by the need to start therapy soon after disclosure of a life-threatening diagnosis. Moreover, the patient is unlikely to fully comprehend the implications of harvesting tissue or having to provide a sample for banking. As previously mentioned, all this has to be individualized, considering cultural and religious beliefs of the patient and his or her family. Lastly, there are difficult decisions that may arise in the event tissue or cells are harvested and cryopreserved, yet the patient does not survive. This highlights the importance of a comprehensive FP program that relies on appropriate ethical and legal expertise.

\section{Expanding the field of fertility preservation}

Rightfully so, the main interest in the field has focused on oncology patients, who represent a vulnerable population at risk for significant problems with fertility. However, as we consider a broader view, other patients can be in similar need for open discussion and FP efforts. This includes children and adolescents with other diagnoses that demand exposure to gonadotoxic agents, such as severe rheumatological or autoimmune diseases, patient with non-malignant conditions that are treated with bone marrow transplantation, and solid organ transplant recipients. Another important group of patients to include are those with disorders of sexual differentiation, who may need to undergo removal of gonadal-discrepant tissue during the difficult process of gender assignment or to minimize the risk of malignant degeneration. The medical, psychological, ethical, and legal issues may seem monumental, yet they appear to be important next steps to further address.

\section{Conclusion}

Fertility preservation is becoming a critical part of comprehensive care of adolescent and children at high risk for future reproductive problems. Oncofertility efforts are leading the way, but the spectrum of care is likely to be amplified in the near future. Urologists with expertise in pediatrics and fertility should consider involvement in this field, as our specialty can add expertise to this exciting initiative.
This paper has been peer-reviewed.

\section{References}

1. Font-Gonzalez A, Mulder RL, Loeffen EA, et al. Fertility preservation in children, adolescents, and young adults with cancer: Quality of clinical practice guidelines and variations in recommendations. Cancer 2016;122:2216-23. htrps://doi.org/10.1002/cncr.30047

2. Survivorship Care for Children. httpswww.cancer.govabout-cancercopingsurvivorshipchild-care. Accessed January 7, 2017.

3. Meistrich ML. Effects of chemotherapy and radiotherapy on spermatogenesis in humans. Fertil Steril 2013;100:1180-6. https://doi.org/10.1016/i.fertnstert.2013.08.010

4. Green DM, Liu W, Kutteh WH, et al. Cumulative alkylating agent exposure and semen parameters in adult survivors of childhood cancer: A report from the St. Jude Lifetime Cohort Study. Lancet Oncol 2014;15:1215-23. https://doi.org/10.1016/S1470-2045(14)70408-5

5. Levine J, Canada A, and Stern CJ. Fertility preservation in adolescents and young adults with cancer. J Clin Oncol 2010;28:4831-41. https://doi.org/10.1200/JC0.2009.22.8312

6. Wasilewski-Masker K, Seidel KD, Leisenring W, et al. Male infertility in long-term survivors of pediatric cancer: A report from the childhood cancer survivor study. J Cancer Surviv 2014;8:437-47. https://doi.org/10.1007/s1 1764-014-0354-6

7. Gupta AA, Donen RM, Sung $L$, et al. Testicular biopsy for fertility preservation in prepubertal boys with cancer: Identifying preferences for procedure and reactions to disclosure practices. J Urol 2016;196:21924. https://doi.org/10.1016/i.juro.2016.02.2967

8. Schover LR. Patient attitudes toward fertility preservation. Pediatr Blood Cancer 2009:53:281-4. https://doi.org/10.1002/pbc.22001

9. Loren AW, Mangu PB, Beck $L N$, et al. Fertility preservation for patients with cancer: American Society of Clinical Oncology clinical practice guideline update. J Clin Oncol 2013;31:2500-10. https://doi.org/10.1200/JC0.2013.49.2678

10. Vindrola-Padros C, Dyer KE, Cyrus J, et al: Healthcare professionals' views on discussing fertility preservation with young cancer patients: A mixed method systematic review of the literature. Psychooncology 2016. [Epub ahead of print] https://doi.org/10.1002/pon.4092

11. Jeruss JS and Woodruff TK. Preservation of fertility in patients with cancer. N Engl I Med 2009;360: 902-11. https://doi.org/10.1056/NEJMra0801454

12. Daudin $M$, Rives $N$, Walschaerts $M$, et al. Sperm cryopreservation in adolescents and young adults with cancer: Results of the French national sperm banking network (CECOS). Fertil Steril 2015;103:478-86. el. https://doi.org/10.1016/j.fertnstert.2014.11.012

13. Keene DJB, Sajiad Y, Makin G, et al. Sperm-banking in the U.K. is feasible in patients 13 years old or older with cancer. J Urol 2012;188:594-7. htrps://doi.org/10.1016/i.juro.2012.04.023

14. Adank $M C$, van Dorp $W$, Smit $M$, et al. Electroejaculation as a method of fertility preservation in boys diagnosed with cancer: A single-centre experience and review of the literature. Fertil Steril 2014;102:199205.el. https://doi.org/10.1016/ifertnstert.2014.03.038

15. Berookhim BM, Mulhall JP. Outcomes of operative sperm retrieval strategies for fertility preservation among males scheduled to undergo cancer treatment. Fertil Steril 2014;101:805-11. https://doi.org/10.1016/iffertnstert.2013.11.122

16. Yokonishi T, Sało T, Komeya M, et al. Offspring production with sperm grown in vitro from cryopreserved testis tissues. Nat Commun 2014;5:4320. https://doi.org/10.1038/ncomms5320

17. Ginsberg JP, Li Y, Carlson CA, et al. Testicular tissue cryopreservation in prepubertal male children: An analysis of parental decision-making. Pediatr Blood Cancer 2014;61:1673-8. https://doi.org/10.1002/ pbc.25078

Correspondence: Dr. Rodrigo L.P. Romao, Division of Pediatric Urology and Pediatric General Surgery, IWK Health Centre, Dalhousie University, Halifax, NS, Canada; rodrigo.romao@dal.ca

Competing interests: The authors report no competing personal or financial interests. 